\title{
High-pressure physical properties of magnesium silicate post-perovskite from $a b$ initio calculations
}

\author{
ZI-JIANG LIU ${ }^{\mathrm{a}, \mathrm{b}, *}$, XIAO-WEI SUN ${ }^{\mathrm{b}}$, CAI-RONG ZHANG ${ }^{\mathrm{c}}$, JIAN-BO HU ${ }^{\mathrm{d}}$, LING-CANG CAI ${ }^{\mathrm{d}}$ \\ and QI-FENG CHEN ${ }^{\mathrm{d}}$

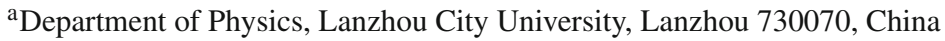 \\ ${ }^{\mathrm{b}}$ School of Mathematics and Physics, Lanzhou Jiaotong University, Lanzhou 730070, China \\ ${ }^{\mathrm{c}}$ State Key Laboratory of Gansu Advanced Non-ferrous Metal Materials, Lanzhou University of Technology, \\ Lanzhou 730050, China \\ ${ }^{\mathrm{d}}$ Laboratory for Shock Wave and Detonation Physics Research, Institute of Fluid Physics, Academy of Engineering \\ Physics, PO Box 919-102, Mianyang 621900, China
}

MS received 1 May 2011

\begin{abstract}
The structure, thermodynamic and elastic properties of magnesium silicate $\left(\mathrm{MgSiO}_{3}\right)$ post-perovskite at high pressure are investigated with quasi-harmonic Debye model and $a b$ initio method based on the density functional theory (DFT). The calculated structural parameters of $\mathrm{MgSiO}_{3}$ post-perovskite are consistent with the available experimental results and the recent theoretical results. The Debye temperature, heat capacity and thermal expansion coefficient at high pressures and temperatures are predicted using the quasi-harmonic Debye model. The elastic constants are calculated using stress-strain relations. A complete elastic tensor of $\mathrm{MgSiO}_{3}$ post-perovskite is determined in the wide pressure range. The calculated elastic anisotropic factors and directional bulk modulus show that $\mathrm{MgSiO}_{3}$ post-perovskite possesses high elastic anisotropy.
\end{abstract}

Keywords. Thermodynamic properties; elastic properties; $\mathrm{MgSiO}_{3}$ post-perovskite.

\section{Introduction}

Studies on the elastic properties of minerals play an indispensable role in the studies of earth's deep interior. The importance of elasticity stems largely from the fact that it is necessary for interpreting seismological information on earth's velocity structure at depth. That is, if one knows the elastic properties of all candidate phases that might exist at depth, then the velocities for any desired assemblage of phases can be calculated (Bass 2008). The high-pressure Pbnm-perovskite polymorph of $\mathrm{MgSiO}_{3}$ is believed to be the most abundant mineral in the earth's lowermost mantle (Knittle and Jeanloz 1987). However, the post-perovskite phase with the $\mathrm{Cmcm} \mathrm{CaIrO}_{3}$ type-structure is recently produced via phase transition from $\mathrm{Pbnm}$ perovskite $\mathrm{MgSiO}_{3}$ at a pressure of 110-125 GPa (Murakami et al 2004; Oganov and Ono 2004). This corresponds roughly to the depth of the $D^{\prime \prime}$ layer near the core-mantle boundary, the validity of such models strongly depends on knowledge of the elastic properties of high-pressure phases of $\mathrm{MgSiO}_{3}$ post-perovskite. The experimental determination of elastic properties at extreme conditions is, however, not an easy task. Up to now, there have not been any measurements reported of the elastic properties of $\mathrm{MgSiO}_{3}$ post-perovskite. Therefore, another approach has been developed based on ab initio quantum

\footnotetext{
*Author for correspondence (liuzj2000@ 126.com)
}

mechanical theory. Ab initio calculations provide the ideal complement to the laboratory approach. Such calculations have true predictive power and can supply critical information including that which is difficult to measure experimentally. The previous theoretical calculations studied the elastic constants of $\mathrm{MgSiO}_{3}$ post-perovskite using the approach (Tsuchiya et al 2004a; Caracas and Cohen 2005). However, many of its elastic tensor is still relatively poorly known. For example, Young's moduli, Poisson's ratio, shear anisotropy, anisotropy of linear bulk modulus and the anisotropy of every symmetry plane and axis etc. These properties play a crucial role in the interpretation of seismic data, and thus have a large influence on our knowledge of the earth's interior.

Determination of thermal properties of $\mathrm{MgSiO}_{3}$ postperovskite is of particular geophysical interest because it can be used to directly constrain the compositional and mineralogical model of the lower mantle by comparing with the observed seismic data. However, it is poorly known even under ambient conditions. For example, its equation of state (EOS) is relatively well known only at $300 \mathrm{~K}$ (Ono et al 2006; Shieh et al 2006; Guignot et al 2007; Shim et al 2008). Wentzcovitch et al (2006) presented the thermoelastic properties of $\mathrm{MgSiO}_{3}$ post-perovskite phase at relevant conditions obtained by $a b$ initio computations within the quasi-harmonic approximation. Tsuchiya et al (2005) used the quasi-harmonic approximation combined with $a b$ initio calculations of the vibrational density of states to compute 
the free energy of $\mathrm{MgSiO}_{3}$ post-perovskite and derive several thermodynamic properties of interest up to $180 \mathrm{GPa}$. In order to further understand the thermodynamics of $\mathrm{MgSiO}_{3}$ postperovskite, present work uses the quasi-harmonic approximation with the Debye model combined with $a b$ initio calculations. Using the method, we obtained the thermodynamic properties of $\mathrm{CaSiO}_{3}$ perovskite at high temperature and high pressure (Liu et al 2007).

In this work, we apply the pseudopotentials planewave method within the generalized gradient approximation (GGA) in the framework of DFT to study elastic properties of $\mathrm{MgSiO}_{3}$ post-perovskite. To further investigate this compound based on $a b$ initio study and the quasi-harmonic Debye model, thermal properties including Debye temperature, heat capacity and thermal expansion are obtained in detail. Our results demonstrate that this method can provide amply reliable predictions for the temperature and pressure dependence of these quantities.

\section{Computational methods}

Quantum first principles calculations for $\mathrm{MgSiO}_{3}$ postperovskite are performed here using the CASTEP code (Segall et al 2002) which uses DFT formalism (Hohenberg and Kohn 1964; Kohn and Sham 1965). A plane-wave basis set is employed to expand the states occupied by the valence electrons, the following valence configurations being taken into account: $2 p^{6} 3 s^{2}$ for $\mathrm{Mg}, 3 s^{2} 3 p^{2}$ for $\mathrm{Si}$ and $2 s^{2} 2 p^{4}$ for O. Vanderbilt-type ultrasoft pseudopotentials (Vanderbilt 1990) are employed to describe the electron ion interactions. The exchange-correlation functional for GGA calculation uses the Perdew-Wang(PW91) (Perdew and Wang 1992) exchange-correlation functional. Integrals in reciprocal space are computed using a $5 \times 5 \times 4$ Monkhorst-Pack grid (Monkhorst and Pack 1976). A geometry optimization procedure is performed on both the unit cell parameters and atomic positions using the Broyden-Fletcher-GoldfarbShanno(BFGS) energy minimizer (Pfrommer et al 1997) to reach a local minimum for the total energy. Convergence thresholds for geometry optimization are $0.5 \times$ $10^{-5} \mathrm{eV} /$ atom for total energy variation, $0 \cdot 01 \mathrm{eV} / \AA$ for maximum force on each atom, 0.02 GPa for the unit cell stress and $5 \times 10^{-4} \AA$ for the maximum atomic displacement. The plane-wave basis set cutoff energy for the calculations is chosen to be $380 \mathrm{eV}$ after convergence studies, and its quality is kept fixed taking into account changes in the unit cell volume during the computer runs.

\section{Results and discussion}

\subsection{Structural properties}

The structural properties of $\mathrm{MgSiO}_{3}$ post-perovskite are calculated by ab initio pseudopotential plane-wave calculations. The total energy is obtained as a function of volume and fitted to the third-order Birch-Murnaghan equation of state (Birch
1978) to obtain the equilibrium volume, $V_{0}$, bulk modulus, $B_{0}$ and its pressure derivative, $B_{0}^{\prime}$. The results are summarized in table 1 , together with some theoretical results (Oganov and Ono 2004; Tsuchiya et al 2004b; Caracas and Cohen 2005) and the available experimental data (Ono et al 2006; Shieh et al 2006; Guignot et al 2007). We find that the equilibrium volume is close to the experimental result, and is in good agreement with the theoretical result. The bulk modulus, $B_{0}$, is consistent with the recent theoretical value (Caracas and Cohen 2005) and the experimental value (Shieh et al 2006). Therefore, excellent agreement strongly supports the choice of pseudopotentials and GGA approximation for the current study.

On the basis of these results, it should be interesting to study the structural properties of $\mathrm{MgSiO}_{3}$ under pressure. The estimation of the zero-temperature transition pressure between the Pbnm perovskite $\mathrm{MgSiO}_{3}$ and $C m c m$ postperovskite $\mathrm{MgSiO}_{3}$ can be obtained from the usual condition of equal enthalpies, in other words, the pressure, $P$, at which enthalpy, $H=E+P V$ of both two phases is the same. The transition pressure from $P b n m$-perovskite polymorph to Cmcm $\mathrm{CaIrO}_{3}$ type-structure of $\mathrm{MgSiO}$ is about $108 \mathrm{GPa}$, which is in good agreement with the in situ X-ray diffraction measurement value of $110 \mathrm{GPa}$ (Murakami et al 2004). This fact will also support the validity of our theoretical predictions of other properties of $\mathrm{MgSiO}_{3}$ post-perovskite under high pressure conditions.

\subsection{Thermodynamic properties}

We choose a Debye-like model as a simple way to take into account the vibrational motion of the lattice. While retaining simplicity of the Debye model, we use a quasi-harmonic approach, making the Debye temperature, $\theta(V)$, dependent upon the volume of the crystal. At each value of $V, \theta(V)$ can be computed accurately in terms of the elastic constants

Table 1. Unit cell volume, bulk modulus and pressure derivatives of $\mathrm{MgSiO}_{3}$ post-perovskite from calculations and measurements.

\begin{tabular}{lccc}
\hline & $V_{0} / \AA^{3}$ & $B_{0} / \mathrm{GPa}$ & $B_{0}^{\prime}$ \\
\hline & Post-perovskite at $0 \mathrm{GPa}$ and $0 \mathrm{~K}$ & \\
This work & $163 \cdot 4$ & 209 & $4 \cdot 4$ \\
LDA[4] & $162 \cdot 9$ & 232 & $4 \cdot 4$ \\
GGA[5] & $163 \cdot 2$ & 203 & $4 \cdot 2$ \\
LDA[22] & $163 \cdot 9$ & 221 & $4 \cdot 2$ \\
Exp.[8] & $162 \cdot 2^{\mathrm{a}}$ & 231 & $4 \cdot 0^{\mathrm{a}}$ \\
Exp.[9] & $164 \cdot 9$ & 219 & $4 \cdot 0^{\mathrm{a}}$ \\
Exp.[10] & $162 \cdot 9^{\mathrm{a}}$ & $237,226,248$ & $4 \cdot 0^{\mathrm{a}}$ \\
& & & \\
This work & $120 \cdot 8$ & 644 & \\
Exp.[7] & $121 \cdot 3$ & 657 & \\
Exp.[9] & $120 \cdot 8$ & 653 & \\
Exp.[25] & $124 \cdot 7$ & 908 & \\
\hline
\end{tabular}

${ }^{\mathrm{a}}$ Fixed. 
(Robie and Edwards 1966). However, a very reasonable alternative is to consider the isotropic approximation. We apply here the quasi-harmonic Debye model, implemented in the pseudo code Gibbs (Blanco et al 2004).

The thermodynamic quantities of $\mathrm{MgSiO}_{3}$ post-perovskite are determined in the temperature range from 0 to $1500 \mathrm{~K}$. The pressure effect is studied up to $150 \mathrm{GPa}$. In order to further check the post-perovskite structure, structural parameters at $300 \mathrm{~K}$ and $125 \mathrm{GPa}$ are calculated using the quasiharmonic Debye model. The results are listed in table 1 along with the available experimental results (Mao et al 2006; Shieh et al 2006; Shim et al 2008). It is seen that the volume is close to the latest experimental data (Shim et al 2008), and is in excellent agreement with the experimental value of Shieh et al (2006). In addition, the bulk modulus is also consistent with the experimental result (Shieh et al 2006).

The Debye temperature is the key quantity in the quasiharmonic Debye model, which correlates with many physical properties of solids, such as specific heat, elastic constants and melting temperature. We calculate dependence of the Debye temperature on pressure and temperature as shown in figure 1. The calculated value of Debye temperature at $0 \mathrm{~K}$ and $0 \mathrm{GPa}$ is $878 \mathrm{~K}$. When the external pressure changes from 0 to $150 \mathrm{GPa}$, the calculated Debye temperature decreases by $18.7 \%$ and $1.4 \%$ at temperatures of 0 and $1500 \mathrm{~K}$, respectively. Additionally, it is seen in figure 1 that the Debye temperature decreases with temperature at a constant pressure and at a constant temperature it follows an almost linear relationship with pressure.

The relationships between heat capacity and temperature at different pressures are shown in figure 2. To confirm the validity of our calculations, comparison of the heat capacity at constant pressure $\left(C_{\mathrm{p}}\right)$ of the perovskite structure between the calculation and the experiment at zero pressure is presented in figure 2(a). We note that the calculated values are in good agreement with the experimental values (Akaogi and Ito 1993). The changing trends of the heat capacity of the perovskite and post-perovskite structures are similar. The post-perovskite structure has a higher value of heat capacity at the same temperature and pressure and the change of heat capacity is much sharper than that of the perovskite structure. Moreover, when $P=0 \mathrm{GPa}$, at sufficiently low temperatures, the heat capacity at constant volume, $C_{\mathrm{V}}$, is proportional to $T^{3}$. This is because only the long wavelength vibration modes of the lattice are populated and these modes could be approximated by treating the lattice as a continuum. However, at high temperatures, $C_{\mathrm{V}}$ converges to a near-constant value, which is in agreement with the law of Petit and Dulong (1819), while $C_{\mathrm{p}}$ increases monotonously with temperature. Figure 2 also indicates that temperature and pressure have opposite influences on the heat capacity and effect of temperature on the heat capacity is more significant than that of pressure. The values of heat capacity become smaller, and its changing trend becomes gentle and the range of agreement with Debye $T^{3}$ law becomes larger with increasing pressure.

Figure 3 shows variation of the thermal expansion coefficient, $\alpha$, with the pressures and temperatures. In order to validate the thermal expansion coefficient, $\alpha$, of the postperovskite structure, we also calculate the thermal expansion coefficient, $\alpha$, of the perovskite structure. It is found that the thermal expansion coefficient, $\alpha$, of the perovskite structure is in accordance with the available measured value (Shim et al 2002) at ambient conditions and fitted values (Mao et al 1991) at zero pressure. From figure 3 we can see that the thermal expansion coefficient, $\alpha$, of the post-perovskite
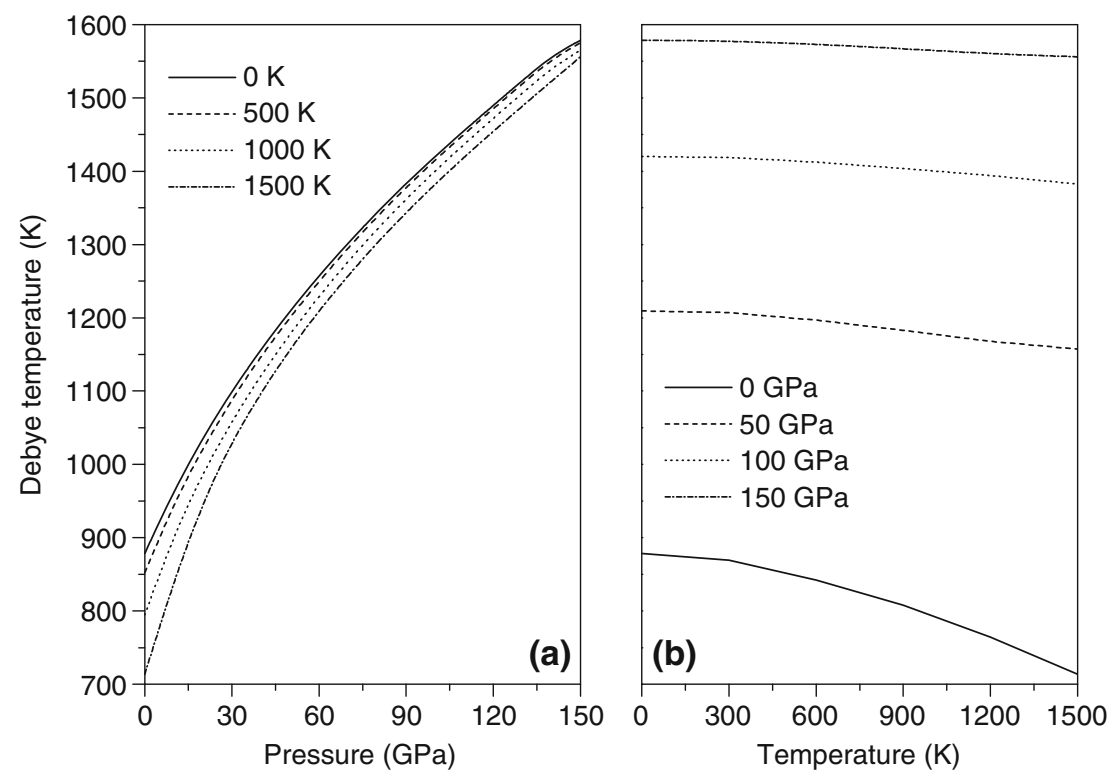

Figure 1. Pressure (a) and temperature (b) dependence of Debye temperature of $\mathrm{MgSiO}_{3}$ post-perovskite. 

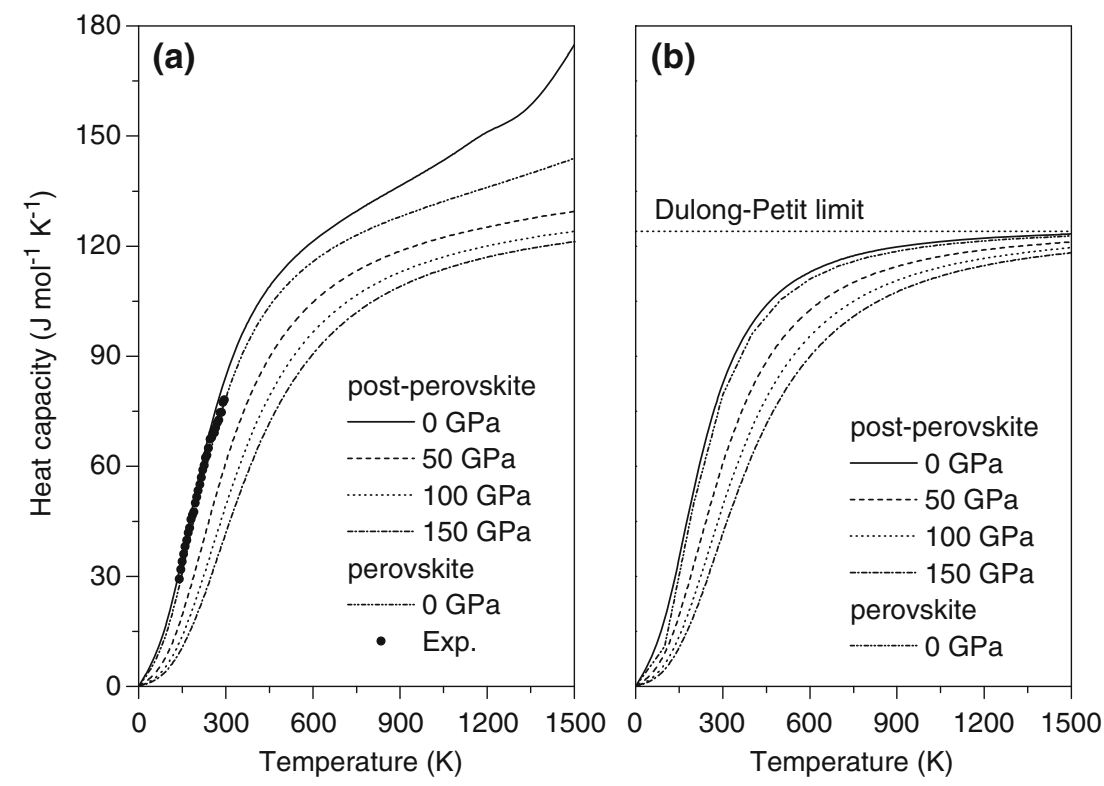

Figure 2. Temperature dependence of constant pressure heat capacity (a) and constant volume heat capacity (b) of $\mathrm{MgSiO}_{3}$ post-perovskite.

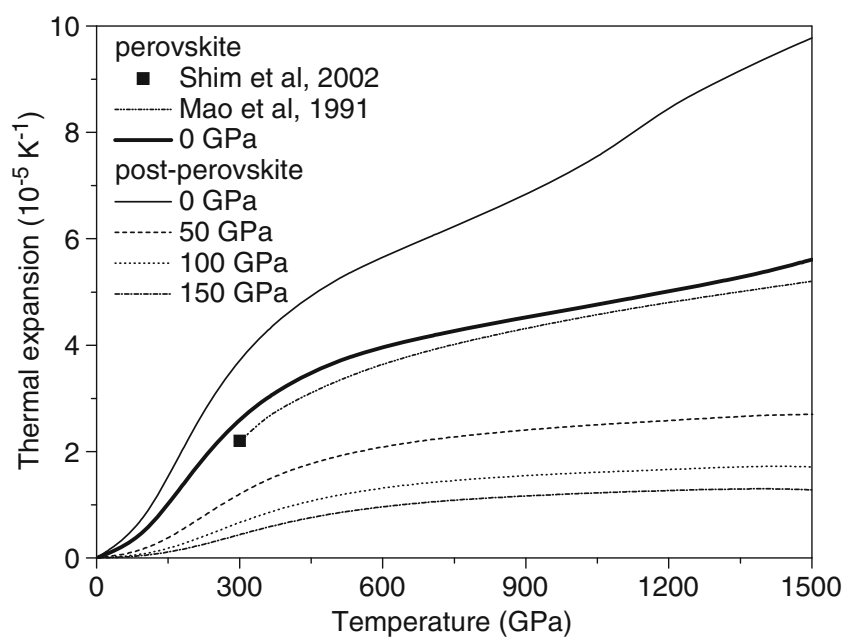

Figure 3. Temperature dependence of thermal expansion coefficient of $\mathrm{MgSiO}_{3}$ post-perovskite.

structure increases with $T^{3}$ at low temperature and gradually increases linearly with the increment of temperatures, and then the increasing trend becomes gentler. The effects of the pressure on the thermal expansion coefficient, $\alpha$, are very small at low temperatures; the effects are increasingly obvious as the temperature increases. As pressure increases, the thermal expansion coefficient, $\alpha$, decreases rapidly and the effects of temperature become less and less pronounced, resulting in linear high temperature behaviour. It is noted that the high temperature dependence of the thermal expansion coefficient, $\alpha$, is not linearly at low pressure, this is an indication of the inadequacy of the quasi-harmonic approximation at high temperatures and low pressures.

\subsection{Elastic properties}

The full elastic constant tensor of $\mathrm{MgSiO}_{3}$ post-perovskite with nine independent components is calculated using stress-strain relations (Karki et al 2001). The calculated elastic constants at $0 \mathrm{GPa}$ and $120 \mathrm{GPa}$ are tabulated in table 2, together with some theoretical results (Oganov and Ono 2004; Tsuchiya et al 2004a; Caracas and Cohen 2005). As LDA tends to overbind the atoms in a crystal, results of LDA is bigger than that of GGA. However, our results are in good agreement with the theoretical results of Oganov and Ono (2004) at $120 \mathrm{GPa}$. We predict high-pressure elastic constants of $\mathrm{MgSiO}_{3}$ post-perovskite because the single-crystal elastic properties of mantle minerals at high pressure are essential for interpreting seismic wave velocities and their lateral variations. Figure 4 shows that the predicted elastic constants increase smoothly and monotonically with increasing pressure. The results are very similar to previous results (Tsuchiya et al 2004a). We find that $c_{22}$ is considerably smaller than $c_{11}$ and $c_{33}$ at relevant pressures, it shows $\mathrm{MgSiO}_{3}$ post-perovskite to be a typical layered mineral. This structure is more compressive in the direction perpendicular to the layers (layers parallel to (010)). In a typical layered structure, lateral shear of layers, along any direction, should offer less resistance than the deformation of the layers themselves, here represented by $c_{55}$. In this structure, the smallest shear elastic constant is $c_{44}$. It expresses the resistance along [001], i.e. to the lateral shift of layers in the direction perpendicular to the octahedral columns.

Based on the predicted elastic constants, the corresponding bulk modulus, $B$ and shear modulus, $G$, can be calculated in terms of Voigt-Reuss-Hill approach (Hill 1952). We calculate the bulk modulus, $B$ and shear modulus, $G$, of $\mathrm{MgSiO}_{3}$ post-perovskite given in table 2. Though the 
Table 2. Elastic constants (in $\mathrm{GPa}$ ) of $\mathrm{MgSiO}_{3}$ post-perovskite at $0 \mathrm{GPa}$ and $120 \mathrm{GPa}$.

\begin{tabular}{lccccccccccc}
\hline & $c_{11}$ & $c_{22}$ & $c_{33}$ & $c_{44}$ & $c_{55}$ & $c_{66}$ & $c_{12}$ & $c_{13}$ & $c_{23}$ & $B$ & $G$ \\
\hline 0 GPa & & & & & & & & & & & \\
This work & 586 & 410 & 454 & 74 & 111 & 83 & 31 & 83 & 94 & 206 & 125 \\
LDA[5] & 649 & 454 & 530 & 79 & 109 & 85 & 40 & 82 & 112 & 232 & 133 \\
LDA[6] & 624 & 433 & 524 & 89 & 110 & 98 & 54 & 82 & 122 & 231 & 136 \\
120 GPa & & & & & & & & & & & \\
This work & 1263 & 923 & 1244 & 297 & 283 & 392 & 387 & 316 & 462 & 637 & 338 \\
GGA[4] & 1252 & 929 & 1233 & 277 & 266 & 408 & 414 & 325 & 478 & 647 & 328 \\
LDA[5] & 1354 & 1007 & 1307 & 290 & 290 & 452 & 417 & 323 & 484 & 677 & 359 \\
LDA[6] & 1308 & 968 & 1298 & 295 & 278 & 439 & 444 & 343 & 507 & 681 & 344 \\
\hline
\end{tabular}
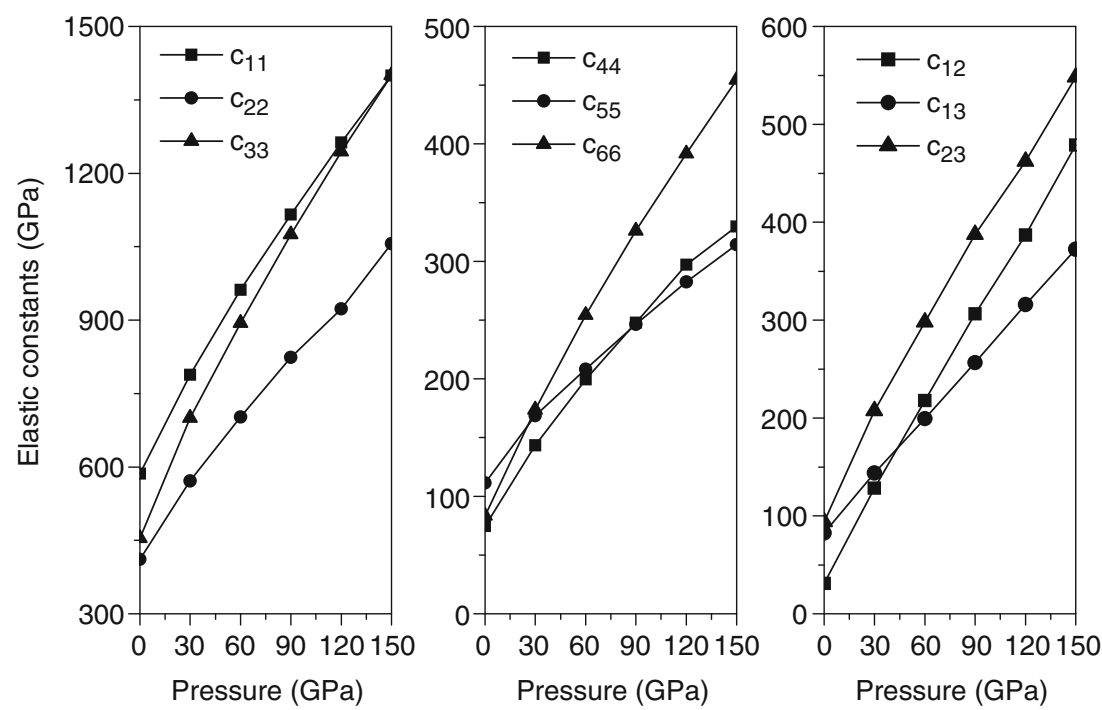

Figure 4. Pressure dependence of elastic constants of $\mathrm{MgSiO}_{3}$ post-perovskite.

calculated bulk modulus is less than that of LDA calculation (Tsuchiya et al 2004a; Caracas and Cohen 2005), they are close to the results of GGA calculation (Oganov and Ono 2004) at $120 \mathrm{GPa}$. Additionally the Young's modulus, $E$ and Poisson ratio, $v$, are calculated using Hill's empirical average:

$$
\begin{aligned}
& E=9 B G /(3 B+G), \\
& \nu=(3 B-2 G) /(6 B+2 G) .
\end{aligned}
$$

The Poisson ratio, $v$, which is associated with the transverse strain under uniaxial press, is obtained as $0 \cdot 25$. The ratio $B / G$ is 1.65 , indicating that it is rather brittle. This is because a high (low) $B / G$ value is associated with ductility (brittleness) and the critical value which separates ductile and brittle materials is about 1.75 (Pugh 1954).

It is known that microcracks are induced in ceramics due to the anisotropy of the coefficient of thermal expansion as well as elastic anisotropy (Tvergaard and Hutchinson 1988). Since all the known crystals are essentially elastically anisotropic, the description of such an anisotropic behaviour is important in both engineering science and crystal physics. For orthorhombic materials, anisotropy arises from shear anisotropy as well as from the anisotropy of the linear bulk modulus. The shear anisotropic factors are

$$
A_{1}=4 c_{44} / c_{11}+c_{33}-2 c_{13},
$$

for the (100) shear planes between the $\langle 011\rangle$ and $\langle 010\rangle$ directions. For the (010) shear planes between $\langle 101\rangle$ and $\langle 001\rangle$ directions, it is

$$
A_{2}=4 c_{55} / c_{22}+c_{33}-2 c_{23},
$$

and for the (001) shear planes between $\langle 110\rangle$ and $\langle 010\rangle$ directions, it is

$$
A_{3}=4 c_{66} / c_{11}+c_{22}-2 c_{12} \text {. }
$$

The deviation of the anisotropic factors from unity is a measure for the elastic anisotropy. The shear anisotropic factors of $\mathrm{MgSiO}_{3}$ post-perovskite are shown in figure 5(a) and we 


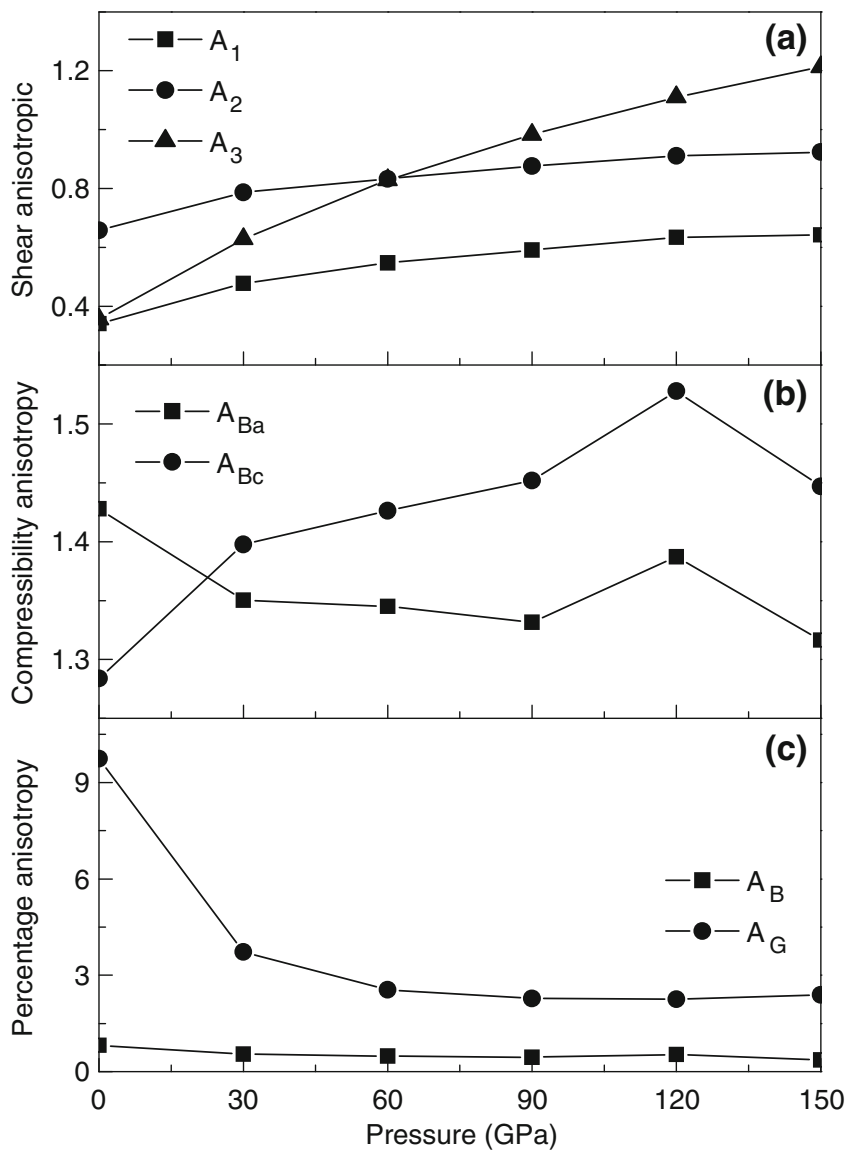

Figure 5. Pressure dependence of elastic anisotropic of $\mathrm{MgSiO}_{3}$ post-perovskite.

find that the factors $A_{1}, A_{2}$ and $A_{3}$ increase with increasing pressure.

In order to investigate the anisotropy arising from the linear bulk modulus, it is useful to calculate the bulk modulus along the crystal axes, defined as (Ravindran et al 1998)

$$
\begin{aligned}
& B_{a}=a \mathrm{~d} P / \mathrm{d} a, \\
& B_{b}=b \mathrm{~d} P / \mathrm{d} b,
\end{aligned}
$$

and

$$
B_{c}=c \mathrm{~d} P / \mathrm{d} c .
$$

The calculated linear bulk modulus along the three directions $a, b, c$ is seen in figure 6 , the linear bulk moduli of the three directions increase with pressure increasing. It indicates that the mechanical anisotropy of $\mathrm{MgSiO}_{3}$ post-perovskite gradually strengthen with increasing pressure. Moreover, it is interesting to note that the directional bulk modulus at high pressure is the largest along the $c$ axis, and the smallest along the $b$ axis, indicating that the compressibility along the $c$ axis is the smallest, while along the $b$ axis is the largest. The compressibility anisotropy of bulk modulus along $a$ and $c$ axes with respect to $b$ axis can then be written as $A_{B_{a}}=B_{a} / B_{b}$

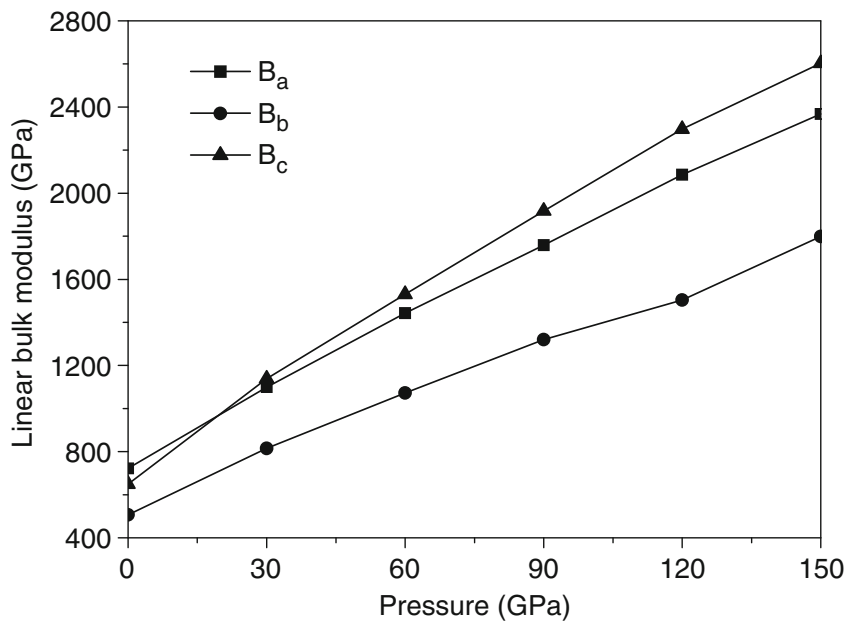

Figure 6. Pressure dependence of linear bulk modulus of $\mathrm{MgSiO}_{3}$ post-perovskite.

and $A_{B_{c}}=B_{c} / B_{b}$. Note that a value of unity for these two fractions represents elastic isotropy and any deviation from 1 provides a measure of degree of anisotropy. The results are noted in figure 5(b). However, in the cubic crystals, the linear bulk modulus is the same for all directions, and the compressibility anisotropy is not applied. To overcome this limitation, Chung and Buessem (1967) introduced a more practical measure of elastic anisotropy for polycrystalline materials where the percentage anisotropy in compressibility and shear is defined as

$$
A_{B}=\left(B_{\mathrm{V}}-B_{\mathrm{R}}\right) /\left(B_{\mathrm{V}}+B_{\mathrm{R}}\right),
$$

and

$$
A_{G}=\left(G_{\mathrm{V}}-G_{\mathrm{R}}\right) /\left(G_{\mathrm{V}}+G_{\mathrm{R}}\right),
$$

respectively, where $B$ and $G$ are the bulk and shear moduli and the subscripts V and R correspond to the Voigt (1928) and Reuss (1929) limits. For these expressions, a value of zero identifies elastic isotropy and a value of $100 \%$ is the largest possible anisotropy. The pressure dependences of the percentage anisotropy in compressibility and shear are shown in figure 5(c). It is found that $A_{B}$ and $A_{G}$ increases with increasing pressure. We also noticed that the percentage bulk modulus anisotropy, $A_{B}$, is smaller than shear modulus anisotropy, $A_{G}$, suggesting that the structure is anisotropic in compressibility.

The anisotropy of the crystal is also measured by $A_{-}$and $A_{+}$coefficients calculated for every symmetry plane and axis. These factors are derived from elastic constants by the following simple relationships (Lau and McCurdy 1998):

$$
\begin{aligned}
& A_{-}^{(010)}=c_{55}\left(c_{11}+2 c_{13}+c_{33}\right) /\left(c_{11} c_{33}-c_{13}^{2}\right), \\
& A_{-}^{(100)}=c_{44}\left(c_{22}+2 c_{23}+c_{33}\right) /\left(c_{22} c_{33}-c_{23}^{2}\right), \\
& A_{-}^{(001)}=c_{66}\left(c_{11}+2 c_{12}+c_{22}\right) /\left(c_{11} c_{22}-c_{12}^{2}\right),
\end{aligned}
$$


Table 3. Anisotropy factors $A_{-}, A_{+}$, respectively for symmetry plane (ijk) and $[i j k]$ symmetry axis with symmetry plane (ijk) as a function of pressure.

\begin{tabular}{|c|c|c|c|c|c|c|c|c|c|}
\hline \multirow[b]{3}{*}{ P/GP } & \multirow{2}{*}{\multicolumn{3}{|c|}{$A_{-}$}} & \multicolumn{6}{|c|}{$A_{+}$} \\
\hline & & & & [100] & [001] & [010] & [001] & [100] & [010] \\
\hline & (010) & (100) & (001) & $(010)$ & (010) & (100) & (100) & $(001)$ & (001) \\
\hline 0 & $0 \cdot 5175$ & 0.4422 & $0 \cdot 3682$ & 0.4424 & $0 \cdot 5992$ & 0.4714 & 0.4144 & $0 \cdot 3004$ & 0.4391 \\
\hline 30 & $0 \cdot 5636$ & 0.6779 & $0 \cdot 6463$ & $0 \cdot 5231$ & $0 \cdot 6060$ & 0.7885 & $0 \cdot 5826$ & $0 \cdot 5260$ & 0.7832 \\
\hline 60 & 0.5728 & 0.8113 & 0.8508 & 0.5465 & 0.5999 & 0.9871 & 0.6693 & $0 \cdot 6838$ & 1.0509 \\
\hline 90 & 0.5876 & 0.9005 & $1 \cdot 0082$ & 0.5737 & $0 \cdot 6018$ & $1 \cdot 1357$ & 0.7203 & 0.8058 & 1.2599 \\
\hline 120 & 0.6029 & 0.9834 & $1 \cdot 1419$ & 0.5969 & $0 \cdot 6090$ & 1.2909 & 0.7602 & $0 \cdot 8946$ & 1.4628 \\
\hline 150 & $0 \cdot 6115$ & 0.9936 & $1 \cdot 242$ & $0 \cdot 6118$ & $0 \cdot 6112$ & $1 \cdot 2973$ & 0.7737 & 0.9875 & 1.5735 \\
\hline
\end{tabular}

$A_{+}^{[100],(010)}=2 c_{55} /\left(c_{11}-c_{13}\right)$

$A_{+}^{[001],(010)}=2 c_{55} /\left(c_{33}-c_{13}\right)$,

$A_{+}^{[010],(100)}=2 c_{44} /\left(c_{22}-c_{23}\right)$,

$A_{+}^{[001],(100)}=2 c_{44} /\left(c_{33}-c_{23}\right)$,

$A_{+}^{[100],(001)}=2 c_{66} /\left(c_{11}-c_{12}\right)$,

$A_{+}^{[010],(001)}=2 c_{66} /\left(c_{22}-c_{12}\right)$.

The calculated anisotropy factors of $\mathrm{MgSiO}_{3}$ post-perovskite are presented in table 3 . One can note systematic and quite substantial (up to $258 \%$ in the case of $A_{+}^{[010](001)}$ ) increase in all anisotropy factors of the crystal. This can indicate an increasing role of anisotropic properties of the magnesium silicate post-perovskite in deep earth conditions, especially when we consider the possibility of large scale alignment of the crystals caused by long time scale laminar flows in the earth's interior.

\section{Conclusions}

We present the results of $a b$ initio calculations for the structure, elastic properties and thermodynamic properties of $\mathrm{MgSiO}_{3}$ post-perovskite. The calculated structural parameters are in agreement with the experimental results and previous calculations. Using the quasi-harmonic Debye model, heat capacity, thermal expansion and Debye temperature are estimated at high temperatures and pressures. Our results demonstrate that this method can provide amply reliable predictions for the temperature and pressure dependence of these quantities. The elastic constants and elastic moduli of $\mathrm{MgSiO}_{3}$ post-perovskite are predicted in this work, but as far as we know, there are no experimental data available for these quantities. Experiments are expected to validate the present numerical data. From the overall behaviour of the elastic constants, it is clear that the structure is quite anisotropic and that anisotropy is strongly pressure dependent.

\section{Acknowledgements}

This work was supported by the National Natural Science Foundation of China under Grant Nos. 11064007 and 11164013, and the New Century Excellent Talents in University under Grant No. NCET-11-0906.

\section{References}

Akaogi M and Ito E 1993 Geophys. Res. Lett. 20105

Bass J D 2008 Phys. Earth Planet. Inter. 170207

Birch F 1978 J. Geophys. Res. 831257

Blanco M A, Francisco E and Luaña V 2004 Comput. Phys. Commun. 15857

Caracas R and Cohen R E 2005 Geophys. Res. Lett. 32 L16310

Chung D H and Buessem W R 1967 J. Appl. Phys. 382010

Guignot N, Andrault D, Morard G, Bolfan-Casanova N and Mezouar M 2007 Earth Planet. Sci. Lett. 256162

Hill R 1952 Proc. Phys. Soc., London A65 349

Hohenberg P and Kohn W 1964 Phys. Rev. 136 B864

Karki B B, Stixrude L and Wentzcovitch R M 2001 Rev. Geophys. 39509

Knittle E and Jeanloz R 1987 Science 235668

Kohn W and Sham L J 1965 Phys. Rev. 140 A1133

Lau K and McCurdy A K 1998 Phys. Rev. B58 8980

Liu Z J, Sun X W, Chen Q F, Cai L C, Wu H Y and Ge S H 2007 J. Phys.: Condens. Matter 19246103

Mao H K, Hemley R J, Fei Y, Shu J F, Chen L C, Jephcoat A P and Wu Y 1991 J. Geophys. Res. 968069

Mao W L, Mao H-K, Prakapenka V B, Shu J and Hemley R J 2006 Geophys. Res. Lett. 33 L12S02

Monkhorst H J and Pack J D 1976 Phys. Rev. B13 5188

Murakami M, Hirose K, Kawamura K, Sata N and Ohishi Y 2004 Science 304855

Oganov A R and Ono S 2004 Nature 430445

Ono S, Kikegawa T and Ohishi Y 2006 Am. Mineral. 91475

Perdew J P and Wang Y 1992 Phys. Rev. B45 13244

Petit A T and Dulong P L 1819 Ann. Chim. Phys. 10395

Pfrommer B G, Cote M, Louie S G and Cohen M L 1997 J. Comput. Phys. 131233

Pugh S F 1954 Philos. Mag. 45823

Ravindran P, Fast L, Korzhavyi P A, Johansson B, Wills J and Eriksson O 1998 J. Appl. Phys. 844891

Reuss A 1929 Z. Angew. Math. Mech. 949 
Robie R A and Edwards J L 1966 J. Appl. Phys. 372659

Segall M D, Lindan P J D, Probert M J, Pickard C J, Hasnip P J, Clark S J and Payne M C 2002 J. Phys.: Condens. Matter 14 2717

Shieh S R, Duffy T S, Kubo A, Shen G, Prakapenka V B, Sata N, Hirose K and Ohishi Y 2006 Proc. Natl. Acad. Sci. USA 1033039

Shim S H, Jeanloz R and Duffy T S 2002 Geophys. Res. Lett. 29 2166

Shim S H, Catalli K, Hustoft J, Kubo A, Prakapenka V B, Caldwell W A and Kunz M 2008 Proc. Natl. Acad. Sci. USA 105 7382
Tsuchiya T, Tsuchiya J, Umemoto K and Wentzcovitch R M 2004a Geophys. Res. Lett. 31 L14603

Tsuchiya T, Tsuchiya J, Umemoto K and Wentzcovitch R M 2004b Earth Planet. Sci. Lett. 224241

Tsuchiya J, Tsuchiya T and Wentzcovitch R M 2005 J. Geophys. Res. 110 B02204

Tvergaard V and Hutchinson J W 1988 J. Am. Ceram. Soc. 71157

Vanderbilt D 1990 Phys. Rev. B41 7892

Voigt W 1928 Lehrbuch de Kristallphysik (Leipzig: Terubner)

Wentzcovitch R M, Tsuchiya T and Tsuchiya J 2006 Proc. Natl. Acad. Sci. USA 103543 